Bio-grafia Escritos sobre la Biología y su Enseñanza.

Edición Extra-Ordinaria. ISSN 2027-1034 P.p 903 - 911

Memorias del VII Encuentro Nacional de Experiencias en la Enseñanza de la Biologia y la Educación Ambiental y II Congreso Nacional de Investigación en la Enseñanza de la Biología

\title{
EL USO DEL GLIFOSATO UNA CUESTION SOCIOCIENTÍFICA COMO CONTRIBUCION AL CUESTIONAMIENTO DE LA INFORMACION
}

\section{GLYPHOSATE USE AS A MATTER SOCIO SCIENTIFIC QUESTIONING CONTRIBUTION TO THE INFORMATION}

\author{
Nidia Yaneth Torres Merchán'.
}

\section{Resumen}

Este trabajo hace parte de una investigación más amplia, sobre los aportes que hacen las cuestiones socio científicas (CSC) al fortalecimiento de las competencias críticas en estudiantes universitarios. En esta comunicación, presentamos los resultados obtenidos a partir de la discusión de una CSC relacionada con el uso del glifosato en la erradicación de cultivos ilícitos, nos enfocaremos en el análisis que los estudiantes hacen frente a las declaraciones de distintos actores que discuten la problemática, los resultados señalan como ese tipo de situaciones contribuye abordar aspectos sociales, éticos, políticos y económicos en la formación de profesores de Ciencias Naturales y Educación Ambiental.

Palabras Clave: Cuestiones socio científicas, pensamiento crítico, glifosato, Educación ambiental.

\section{Abstract}

This work is part of a wider investigation, in to the contributions made socio scientific issues to streng then the critical skills in college students. In this paper we present, the results obtained from the discussion of as cientific social issue related to the use of glyphosate on the eradication of illicit crops, we will focus on the analysis that students face different actors statements discussing the problem the results show, as such situations helps address social, ethical, political and economic in the training of teachers of Natural Science and Environmental Education.

\section{Introducción}

El uso del glifosato en la erradicación de los cultivos ilícitos en Colombia, ha tomado el interés de los últimos gobiernos quienes han implementado políticas de erradicación como parte de la agenda de lucha contra el narcotráfico; esta

\footnotetext{
${ }^{1}$ Facultad de Educación. Universidad Pedagógica y Tecnológica de Colombia.nidia.torres@uptc.edu.co
} 
Bio-grafia Escritos sobre la Biología y su Enseñanza.

Edición Extra-Ordinaria. ISSN 2027-1034 P.p 903 - 911

Memorias del VII Encuentro Nacional de Experiencias en la Enseñanza de la Biología y la Educación Ambiental y II Congreso Nacional de Investigación en la Enseñanza de la Biología

situación ha generado intensos debates, considerando que algunos alegan secuelas negativas, y otros efectos positivos como medida de erradicación del cultivo de la coca; este escenario ha sido abordado desde el enfoque de las cuestiones socio científicas (CSC) por ser una situación controversial que permite abordar aspectos sociales, éticos, científicos, que se constituye en un aporte importante al desarrollo de competencias críticas. (Solbes y Torres 2012).

Por ello, se presenta en esta comunicación el abordaje de una situación controversial relacionada con el uso del glifosato que puede dar aportes importantes a estar informado sobre el tema, no limitarse al discurso dominante y conocer posturas alternativas, cuestionar la validez de los argumentos, rechazando conclusiones no basadas en pruebas, detectar falacias argumentativas y evaluar la credibilidad de las fuentes teniendo en cuenta los intereses subyacentes; lo anterior ha sido pensada como una competencia critica con contribuciones al desarrollo del pensamiento crítico a partir del uso de las CSC en el marco de la investigación que adelanta la autora de este trabajo.

En la época actual hay una sociedad más tecnificada que pocas veces se pregunta el porqué de las cosas, se acepta fácilmente información de la publicidad, sin cuestionamiento; un ejemplo es el efecto milagroso de los productos cosméticos y los artefactos tecnológicos; estos aspectos permiten que desde el abordaje de las CSC se reconozca la necesidad de verificar, analizar y cuestionar muchos de los fundamentos que creemos como verdaderos, esto se constituye en un aporte interesante en la formación inicial de maestros en Ciencias para mantener perspectivas de formación frente a la información que diariamente circula en los medios de comunicación. (Torres, 2011)

El cuestionamiento constante de la información, permite elegir mejor, vislumbrar las falacias del mercado, la política de los medios de comunicación; mientras que una actitud acrítica no promueve los elementos necesarios para tomar decisiones fundamentadas que llevan actuar en consecuencia con desigualdades e injusticias sociales. En este sentido, es importante posibilitar espacios para el cuestionamiento de la información en las clases que permita emitir juicios con conocimiento de los temas, razón y justificación de los hechos.

En este orden de ideas, este trabajo tiene el objetivo principal de analizar la manera que los estudiantes cuestionan la información abordando una CSC relacionada con el uso del glifosato en la erradicación de cultivos ilícitos en Colombia. 
Bio-grafia Escritos sobre la Biología y su Enseñanza.

Edición Extra-Ordinaria. ISSN 2027-1034 P.p 903 - 911

Memorias del VII Encuentro Nacional de Experiencias en la Enseñanza de la Biología y la Educación Ambiental y II Congreso Nacional de Investigación en la Enseñanza de la Biología

\section{Marco Teórico}

Varias investigaciones han considerado el uso de las CSC en las clases de ciencias y se destaca su importancia en la formación ciudadana como un objetivo central de la enseñanza de la ciencias (Ratcliffe y Grace, 2003; Abd-el-Khalick, 2003; Martinez, 2010; Díaz y Jiménez, 2012). Por ejemplo estudios de Sadler et al (2004), destacan que este tipo de experiencia educativa ayuda a los estudiantes a comprender situaciones sociales, los actos humanos y las cuestiones que por ellas son producidas, desde esta perspectiva, esta autora señala que es necesario que la escuela forme los conocimientos científicos mínimos para que los ciudadanos tengan las condiciones de juzgar el contexto científico y tomen decisiones fundamentadas.

Jiménez-Aleixandre (2010) resalta la importancia que ofrecen las CSC en la autonomía de los estudiantes y el empoderamiento, afirma que es una estrategia para el desarrollo de una opinión independiente, que permite reconocer la existencia de distintos puntos de vista.

Desde los referentes anteriores se presenta en este trabajo como el uso de Glifosato puede ser asumida como una CSC por un lado investigaciones de Andres Carrasco, Rafael Paramo, Jorge Kaczewer, Gilles- Eric Seralin, muestran que el glifosato interfiere en el metabolismo enzimático de todos los vertebrados y produce malformaciones congénitas. En argentina, el glifosato es cuestionado por su uso en cultivos de soya transgénica, mientras que en Colombia, es una situación que pone por un lado las políticas del gobierno frente a la erradicación de cultivos y su efecto en la salud de las personas y los ecosistemas.

Esta CSC permite abordar otros aspectos sociales como la falta de políticas agrarias que responda a las demandas campesinas, en lo referente al mantenimiento de una vida en condiciones mínimas de dignidad, la producción de coca se convierte "en la única alternativa para los campesinos frente a la crisis de la agricultura, dado que este sector ha sido la principal víctima del proceso de globalización (El plan Colombia, 2001).

De la misma manera estudios de la Universidad Nacional de Colombia por el grupo de investigación de Toxicología acuática muestran como el glifosato en concentraciones mínimas producen alteraciones en la bioquímica de los organismos. El estudio realizado con cachama blanca, permitió determinar que el uso de glifosato controlado en concentraciones de 5 y $15 \mathrm{ppm}$ produce la elevación de enzimas del plasma de la sangre y se evidencia los primeros estadios al daño celular.

Estos aspectos permiten asumir el uso del glifosato como CSC para generar espacios de discusión y reflexión crítica en la formación de profesores en 
Bio-grafia Escritos sobre la Biología y su Enseñanza.

Edición Extra-Ordinaria. ISSN 2027-1034 P.p 903 - 911

Memorias del VII Encuentro Nacional de Experiencias en la Enseñanza de la Biología y la Educación Ambiental y II Congreso Nacional de Investigación en la Enseñanza de la Biología

ciencias, donde se tomen posiciones con fundamentos a favor o en contra de los hechos presentados, permite mostrar los conocimientos científicos de una forma contextualizada; puede ser desde vivencias y referencias académicas de los alumnos o de simplemente desde información divulgada por los medios de comunicación.

\section{Metodología}

La estructuración de la actividad inicia con una descripción acerca del uso del glifosato en Colombia, como resultado de la inclusión de planes de gobierno contra el narcotráfico; posteriormente se presenta una situación hipotética que inicia con la denuncia por parte de un campesino acerca de los efectos del glifosato en su región. Este campesino pide a los representantes de la industria que expliquen porque se están presentando tales anomalías y al gobierno pregunta ¿Qué ha realizado para subsanar los daños ocasionados?

Luego se presentan algunos fragmentos de las declaraciones de los distintos actores (Empresarios, campesinos, científicos, gobierno) con el fin de que los estudiantes analicen estas declaraciones y evalúen su veracidad.

En esta actividad participaron 16 grupos de 3 estudiantes y 2 grupos de 4 estudiantes, es decir que la información en las distintas categorías es producto del análisis de 18 trabajos elaborados por los grupos mencionados.

Para el análisis de la información arrojada por la actividad, se establecieron categorías de análisis considerando las respuestas de los grupos de trabajo se realiza una descripción textual de las respuestas dadas por los estudiantes, que permite identificar la manera como lo estudiantes cuestionan la declaraciones de distintos actores que emiten declaraciones respecto al uso del Glifosato en la erradicación de cultivos ilícito en Colombia.

\section{Resultados Y Discusión}

La siguiente pregunta propone que los estudiantes valoren críticamente cada una de las declaraciones propuestas por los actores que discuten el uso del glifosato en la erradicación de los cultivos ilícitos. Para ello, mostramos las declaraciones de los actores que evidencian la forma de opinión de cada uno: 
Bio-grafía Escritos sobre la Biologia y su Enseñanza.

Edición Extra-Ordinaria. ISSN 2027-1034 P.p 903 - 911

Memorias del VII Encuentro Nacional de Experiencias en la Enseñanza de la Biología y la Educación Ambiental y II Congreso Nacional de Investigación en la Enseñanza de la Biología

Empresario

- De acuerdo a las recomendaciones de nuestra empresa esta [sustancia química] no representa daño alguno si se respetan las tasas de aplicación para plantas leñosas como la coca, y de acuerdo a estudios por otras entidades se observa que la mezcla está clasificada como ligeramente toxica; pero considerando la situación presentada por la comunidad, se puede indicar que los encargados de realizar las respectivas mezclas están utilizando cantidades mayores a las realizar las respectivas mezclas están utizando cantidades mayores a las efectos que tiene cualquier tipo de producto que se utilice. acerca de los posibles

\section{Gobierno}

- El plan de gobierno no puede permitir que se continúe con la implementación de cultivos ilicitos; por lo tanto si la comunidad no quiere que se siga utilizando este quimicos deberia también comprometerse a no sembrar la coca y a denunciar a las personas que cometen este delito, sin embargo son pocas las denuncias que hasta el momento se han presentado pero dada la gravedad de la situación nos comprometemos a buscar otras alternativas de erradicación tales como la erradicación manual la creación de concejos comunales $y$ otras alternativas de economía.

\section{Campesino}

- Nosotros no sabemos leer y no somos los que lo utilizamos, nuestra comunidad estaba bien hasta cuando ustedes empezaron a utilizar este veneno; además nos gustaría saber si la información que ustedes acaban de dar solo corresponde a un estudio o se han realizado estudios más seguidos a partir de la aplicación de dicho producto.

\section{Cientificos}

- Ct. Las propiedades físicas, químicas y biológicas del glifosato y del adyuvante (Cosmo-Flux) añadido a la mezcla de aspersión se caracterizaron mediante la literatura científica y por medio de nuevos estudios adelantados específicamente para esta evaluación del riesgo. El glifosato no es muy móvil en el ambiente y se une rápida y fuertemente al entrar en contacto con el suelo y con sedimentos acuáticos. El glifosato tiene una actividad biológica de corta duración en suelos y agua, no se biomagnifica ni se mueve a lo largo de la cadena alimenticia, y no se filtra a las aguas subterráneas desde el suelo. La toxicidad del glifosato ha sido rigurosamente evaluada en muchas partes y en la literatura publicada. El glifosato tiene baja toxicidad en otros organismos que no son el objetivo, excepto para las plantas verdes. Se considera de baja toxicidad en forma aguda o crónica; no es carcinogénico, ni mutagénico y tampoco es lesivo para la reproducción. Con respecto a los humanos, no se le considera nocivo, excepto por la posibilidad de irritación ocular transitoria y probablemente, cutánea (con recuperación de ambas).

A partir de lo anterior, se les plantea la siguiente pregunta:

b. ¿Cómo valoras las declaraciones de cado uno de los actores? Justifica su respuesta.

\begin{tabular}{|l|l|l|l|}
\hline $\begin{array}{l}\text { Cada uno declara de de } \\
\text { acuerdo a sus } \\
\text { conocimientos intereses y } \\
\text { necesidades. }\end{array}$ & $\begin{array}{l}\text { Los que tienen el } \\
\text { poder } \\
\text { argumentan más } \\
\text { dejando a los } \\
\text { campesinos } \\
\text { sometidos. }\end{array}$ & $\begin{array}{l}\text { Hace falta una } \\
\text { toma de } \\
\text { conciencia de los } \\
\text { distintos actores. }\end{array}$ & $\begin{array}{l}\text { Todos } \\
\text { dicen } \\
\text { mentiras a } \\
\text { excepción } \\
\text { de los } \\
\text { campesino } \\
\text { s. }\end{array}$ \\
\hline $\begin{array}{l}\text { G1, G3, G4, G2,15, G11,G16 } \\
\text { G8,G10,G12,G13, } \\
\text { G14,G18 }\end{array}$ & G5, G7,G9 & G6,G17 \\
\end{tabular}

La tabla anterior permite inferir que la categoría con mayor número de grupos es la primera que indica que las declaraciones de cada uno de los actores es producto de los conocimientos que poseen.

G8. Cada uno habla desde sus conocimientos, por ejemplo Don Juan por el daño que está causando el glifosato en sus cosechas, el empresario manifiesta que el glifosato es efectivo respetando las cantidades señaladas, el gobierno debe cumplir con planes políticos y el científico utiliza el lenguaje de la ciencia para decir que el glifosato no es toxico. 
Bio-grafia Escritos sobre la Biología y su Enseñanza.

Edición Extra-Ordinaria. ISSN 2027-1034 P.p 903 - 911

Memorias del VII Encuentro Nacional de Experiencias en la Enseñanza de la

Biología y la Educación Ambiental y II Congreso Nacional de Investigación en la Enseñanza de la Biología

Aunque en la declaración anterior, el grupo manifiesta que los actores hablan desde sus conocimientos es evidente que lo hacen desde sus intereses, en este caso dice el gobierno debe cumplir con los planes políticos, el campesino habla desde su realidad, es decir, los cambios que han visto en sus cosechas y el científico utiliza en la declaración términos como: el glifosato no es muy móvil en el ambiente, el glifosato tiene una actividad biológica de corta duración que no se biomagnifica.

Otros grupos G2, G11, G15, G16 manifiestan que se observa unas opiniones de dominación del gobierno y de los empresarios frente a las del campesino.

G16. Todos hablan defendiendo el glifosato pero las personas más afectadas por este hecho inaceptable son los campesinos, por el efecto que han sufrido sus cosechas.

Los grupos G4, G10, G14 y G18 señalan que cada actor habla de acuerdo a sus intereses y justifica su acción desde ese punto. En el caso del empresario y el científico en su declaración se observa un traslado de culpabilidad a las personas que realizan la mezcla, afirmando que estas personas utilizan cantidades mayores a las permitidas, esto hace que los grupos manifiestan su total desacuerdo.

G14. Por ejemplo, en el empresario para él es conveniente hablar del químico diciendo que la dosis que se está aplicando no es la debida que por eso se están presentando problemas por la utilización de este químico, si los campesinos no cuentan con una educación como se van a informar de los efectos realmente y se nota que hay intereses de por medio.

En el caso del grupo G14 se observa como hacen un señalamiento a la educación convertida en una posibilidad de élite en la que los campesinos tienen poca posibilidad de acceso, esta apreciación pone en discusión aspectos sociales en relación a desigualdades.

Los grupos G5, G7 y G9 manifiestan que por interés individual se olvidan de los efectos del glifosato en la población. La declaración del G5 recalca la necesidad de buscar los actores involucrados soluciones conjuntas.

G5.Desde nuestro punto de vista nos parece que hace falta una toma de conciencia por cada uno de los actores, pues cada uno en su afán de demostrar de los que es capaz, se olvidaron que más que tratar de erradicar un cultivo hay una población que merece el debido cuidado y la debida atención para que no salga afectado de ninguna manera, por eso no es suficiente decir que ellos cumplen con su trabajo y que se están presentando algunos problemas con este químico es porque la persona que lleva a cabo el proceso no lo hace bien, es simplemente tratar de buscar otras soluciones y no lavarnos las manos 
Bio-grafia Escritos sobre la Biología y su Enseñanza.

Edición Extra-Ordinaria. ISSN 2027-1034 P.p 903 - 911

Memorias del VII Encuentro Nacional de Experiencias en la Enseñanza de la

Biología y la Educación Ambiental y II Congreso Nacional de Investigación en la Enseñanza de la Biología

con los otros, pues si cada una de estas personas crítica y justifica su trabajo por su rango y sus creencias siempre buscando beneficio realmente no se está buscando dar una solución a una problemática sino que se está logrando agrandar el problema pues una vez demostradas las causas secundarias de este químico hay que buscar una solución como dicen la cura es más grave que la enfermedad.

Otra actividad que fue abordada, hace referencia al tipo de cuestionamientos que los estudiantes efectuarían a los actores. En relación a las preguntas, los estudiantes proponen al uso de evidencias que demuestren que efectivamente el glifosato es el causante de ese tipo de afectaciones, de igual forma hacen énfasis en la necesidad de buscar alternativas sanas para erradicar los cultivos de coca y destacan la importancia de mantenerse informado frente a lo que se usa y se consume.

A los empresarios les reclaman la necesidad de informar a la comunidad el uso adecuado de los productos fabricados, solicitan el respaldo de estudios serios que manifiesten los efectos reales del glifosato, cuestionan la falta de interés de las industrias que conocedoras de los efectos del glifosato siguen comercializándolo.

Al gobierno, piden el uso de otros tipos de planes para la erradicación de cultivos de coca, la necesidad de comprometerse más con los campesinos, el desarrollo de proyecto de conservación de ecosistema, se resalta la necesidad de hacer estudios serios acerca de los efectos del glifosato antes de autorizar su uso, apoyo y claridad en políticas agrarias.

Por último, a los científicos les cuestionan la fabricación de productos tóxicos, señalan que se deberían realizar estudios que produzcan sustancias no toxicas sin implicaciones negativas en la población. De la misma manera, realizan preguntas relacionadas con el efecto de la sustancia como por ejemplo que pasa si se elimina el Cosmo flux o si cambia la dosis de fumigación. Preguntan acerca de la existencia de intereses económicos pagados por el gobierno o empresas como Monsanto.

\section{Conclusiones}

El uso del glifosato se constituye en una CSC que permite espacios de discusión y cuestionamiento de declaraciones por distintos actores sociales, evoca a informase sobre el tema, no limitarse a miradas unidireccionales, conocer posturas alternativas que permiten valorar y reflexionar situaciones sociales desde escenarios de formación inicial en docentes.

Se reconoce el uso del glifosato como una situación controversial; es decir, una situación que pone en discusión, las políticas del gobierno frente a la erradicación 
Bio-grafia Escritos sobre la Biología y su Enseñanza.

Edición Extra-Ordinaria. ISSN 2027-1034 P.p 903 - 911

Memorias del VII Encuentro Nacional de Experiencias en la Enseñanza de la Biología y la Educación Ambiental y II Congreso Nacional de Investigación en la Enseñanza de la Biología

de cultivos ilícitos y sus implicaciones en los habitantes y los ecosistema de las zonas que están siendo fumigadas y se promueve la manifestación de puntos de vistas propios sobre cuestiones que necesariamente abordan como el desplazamiento de la campesinos, la toxicidad del glifosato, el interés económico de las industrias, afectaciones ambientales y sociales; estos aspectos se constituye en una posibilidad para incrementar los niveles de comunicaciones entre los mismos estudiantes para comprender situaciones sociales.

Se observó que el abordaje de las CSC puede ser una oportunidad para establecer relaciones entre aspectos científicos, éticos, sociales, económicos, ambientales; sin embargo, los participantes tienden a explicar la situación de manera separada, por lo que este tipo de situaciones permiten evidenciar que la articulación de los conceptos científicos a situaciones reales es un mecanismo de dificultad para los estudiantes; lo cual permite pensar en estrategias didácticas que no atomicen los contenidos sino que le apuesten a la integralidad como oportunidad para manifestar estrategias creativas en la enseñanza de las ciencias.

\section{Referencias Bibliográficas}

Abd-el-khalick, F. (2003).Socioscientific issues in pre-college science classrooms: theprimacy of learners' epistemological orientations and views of nature of science In: ZEIDLER, D. (Org.). The role of moral reasoning on socioscientific issues and discourse in science education. The Netherlands: Kluwer Academic Publishers, p. 41-61.

Díaz, N y Jiménez, M. (2012). Las controversias sociocientíficas: temáticas e importancia para la educación científica. Revista Eureka sobre Enseñanza y Divulgación de lasCiencias 9(1), 54-70.

EL PLAN COLOMBIA". Visiones Críticas desde el Congreso, La Academia y Las Regiones. En Revista Gaceta. Septiembre 2001. No 3.

Jiménez-Aleixandre, M. P.(2010). 10 ideas clave. Competencias en argumentación y uso de pruebas.Barcelona: Graó.

Martínez, L. (2010). A abordagem de questõessociocientíficasnaformação continuadade profesores de Ciências: contribuições e dificuldades. Tesis de Doutorado: Faculdade de Ciências da Universidade Estadual Paulista, Bauru. 
Bio-grafía Escritos sobre la Biologia y su Enseñanza.

Edición Extra-Ordinaria. ISSN 2027-1034 P.p 903 - 911

Memorias del VII Encuentro Nacional de Experiencias en la Enseñanza de la

Biología y la Educación Ambiental y II Congreso Nacional de Investigación en la Enseñanza de la Biología

Sadler, T.; Chambers, F.; Zeidler, D. (2004) Students conceptualizations of thenature of science in response to a socioscientific issue. International journal of Science Education, 26, 387-410.

Solbes, J y Torres, N. (2012). Análisis de las concepciones y las competencias de pensamiento crítico desde el abordaje de las cuestiones sociocientíficas: un estudio en el ámbito universitario. Didáctica de las ciencias experimentales y sociales.26, 247-269.

Torres, N. (2011): Las Cuestiones Sociocientificas como Estrategia Didáctica para el Desarrollo del Trabajo Colaborativo en el Aula de Clase. Revista "Entre Comillas", ISSN: 0-124-5872 n. 14, p. 47-58. 